La Revue

des Droits

de l'Homme

\section{La Revue des droits de l'homme}

Revue du Centre de recherches et d'études sur les droits fondamentaux

Actualités Droits-Libertés | 2011

Liberté de circulation des citoyens européens (art. 16 et 28 de la directive 2004/38/ce)

\title{
Protection renforcée contre l'éloignement et « raisons impérieuses de sécurité publique "
}

Liberté de circulation des citoyens européens (art. 16 et 28 de la directive 2004/38/ce)

\section{Myriam Benlolo Carabot}

\section{OpenEdition \\ Journals}

Édition électronique

URL : http://journals.openedition.org/revdh/3708

DOI : $10.4000 /$ revdh.3708

ISSN : 2264-119X

Éditeur

Centre de recherches et d'études sur les droits fondamentaux

Référence électronique

Myriam Benlolo Carabot, « Protection renforcée contre l'éloignement et « raisons impérieuses de

sécurité publique»" ", La Revue des droits de l'homme [En ligne], Actualités Droits-Libertés, mis en ligne le 06 janvier 2011, consulté le 19 avril 2019. URL : http://journals.openedition.org/revdh/3708 ; DOI :

10.4000/revdh.3708

Ce document a été généré automatiquement le 19 avril 2019

Tous droits réservés 

de sécurité publique»

Liberté de circulation des citoyens européens (art. 16 et 28 de la directive 2004/38/ce)

\author{
Myriam Benlolo Carabot
}

1 M. Tsakouridis, de nationalité grecque, est né en Allemagne en 1978, a toujours vécu et a effectué toute sa scolarité sur le territoire de cet Etat membre. Condamné pour plusieurs délits entre 1998 et 2002, il a travaillé en Grèce quelques mois en 2004, puis pendant seize mois entre 2005 et 2007. Il ne revient en Allemagne que le 19 mars 2007, suite à un mandat d'arrêt émis par les autorités allemandes fin 2005. Il est condamné en août 2007 du chef de commerce illégal en bande organisée de stupéfiants en grandes quantités, à huit reprises, à une peine de six ans et six mois d'emprisonnement. La perte de son droit d'entrer et de séjourner sur le territoire allemand est constatée en août 2008, par une décision qui sera annulée par le tribunal de Stuttgart le 24 novembre 2008 au motif qu'une condamnation pénale ne suffit pas en soi pour fonder la déchéance du droit d'entrer et de séjourner d'un citoyen de l'Union, et que dans le cas de l'intéressé, qui a séjourné plus de dix ans sur le territoire allemand, la déchéance du droit d'entrer et de séjourner ne peut être constatée qu'en présence de raisons impérieuses de sécurité publique, du fait des exigences posées par l'article 6, paragraphe 5, de la loi allemande sur la libre circulation des citoyens de l'Union, qui transpose l'article 28, paragraphe 3, de la directive 2004/38/CE relative au droit des citoyens de l'Union et des membres de leur famille de circuler et de séjourner librement sur le territoire des Etats membres. Saisi d'un appel contre l'arrêt, le Verwaltungsgerichtshof Baden-Württemberg a posé plusieurs questions préjudicielles à la Cour de justice de l'UE, relatives à l'interprétation de l'article 28, paragraphe 3 , de la directive. Plus précisément, la Cour doit se prononcer sur le régime de protection renforcée mis en œuvre par cette disposition, qui énonce qu'une 
décision d'éloignement "ne peut être prise à l'encontre des citoyens de l'Union, quelle que soit leur nationalité, à moins que la décision ne se fonde sur des raisons impérieuses de sécurité publique définies par les Etats membres, si ceux-ci : a) ont séjourné dans l'Etat membre d'accueil pendant les dix années précédentes ». Reste à savoir d'une part, si et dans quelle mesure des absences du territoire pendant cette période privent l'individu de la protection renforcée, et d'autre part, si et dans quelle mesure la criminalité liée au trafic de stupéfiants en bande organisée est susceptible de relever de la notion de "raisons impérieuses de sécurité publique».

2 La Cour n'a de cesse de montrer dans son raisonnement la spécificité de la situation des citoyens de l'Union qui ont acquis un droit de séjour permanent dans leur Etat membre d'accueil et qui, de surcroît, peuvent justifier d'une durée de résidence de dix ans dans celui-ci au moment où la décision d'éloignement est prise par les autorités nationales. Estimant que dans ce dernier cas, la directive "renforce considérablement» la protection contre les mesures d'éloignement (point 28), la Cour juge que pour déterminer dans quelle mesure des absences du territoire peuvent priver l'individu de ce régime particulier, « il y a lieu d'effectuer une appréciation globale de la situation de l'intéressé chaque fois au moment précis où se pose la question de l'éloignement » (point 32). Parce que la directive 2004/38 met en place un régime de protection à l'encontre des mesures d'éloignement qui est fondé sur le degré d'intégration des personnes concernées dans l'Etat membre d'accueil (point 25), il faut en fait que les autorités nationales vérifient, en prenant en compte la durée des absences, leur fréquence et leurs motivations, si ces absences " impliquent le déplacement vers un autre Etat du centre des intérêts personnels, familiaux ou professionnels de l'intéressé » (point 33). La Cour reprend ici la définition de la résidence qu'elle a pu proposer dans d'autres domaines du droit de l'Union, notamment celui des règlements de coordination des régimes de sécurité sociale (CJCE, 12 juillet 1973, Angenieux, aff. 13/73, Rec., p. 950, point 29 ; CJCE, 17 février 1977, Di Paolo, aff. 76/76, Rec., p. 325, point 17), ou de la fonction publique (CJCE, 15 septembre 1994, Pedro Magdalena Fernandez, aff. C-452/93, Rec., pp. I-4308-4309, point 22). En transposant cette définition dans le domaine de la citoyenneté de l'Union, la Cour confirme la construction encore hésitante d'une citoyenneté de résidence, fondée sur l'intégration du ressortissant dans son Etat d'accueil (sur le lien entre le citoyen de l'Union et le territoire de l'Etat d'accueil et le «degré d'intégration » du citoyen, voir, entre autres, les arrêts de la CJCE du 15 mars 2005, Bidar, aff. C-209/03, Rec., p. I-2119, et du 18 novembre 2008, Förster, aff. C-158/07, Rec., p. I-8507).

3 Si la juridiction nationale devait conclure au vu de ces indications que l'intéressé peut se prévaloir de la protection renforcée prévue par l'article 28 , paragraphe 3 , de la directive 2004/38, elle devrait ensuite examiner si la décision se fonde sur des « raisons impérieuses de sécurité publique» au sens de cette même disposition. Rappelant qu'elle peut dans sa réponse prendre en considération des normes du droit de l'Union auxquelles le juge national n'a pas fait référence dans ses questions préjudicielles, la Cour va souligner avec une insistance tout à fait singulière le caractère très exceptionnel d'une mesure d'éloignement du territoire prise dans le cas d'un individu pouvant se prévaloir de la protection renforcée. La notion de "raisons impérieuses de sécurité publique» est " considérablement plus stricte» que celle de "motifs graves» au sens du paragraphe 2 de l'article 28 , ce qui veut donc dire que les mesures d'éloignement fondées sur le paragraphe 3 ne peuvent être limitées qu'à des «circonstances exceptionnelles» (point 40). L'atteinte à la sécurité publique doit présenter " un degré de gravité particulièrement élevé » 
(point 41), la menace doit être d'une "exceptionnelle gravité»(point 49). Le degré d'intégration de l'intéressé, la solidité de ses liens familiaux, culturels et sociaux avec l'Etat membre d'accueil, justifient un niveau de protection extrêmement élevé, dans lequel la Cour n'accepte finalement que peu la marge de manœuvre nécessairement laissée à l'Etat pour définir les contours de l'ordre public. Alors que l'Avocat général avait insisté dans ses conclusions sur celle-ci (points 69 à 72), la Cour n'y fait quant à elle aucune référence : selon le juge de l'Union, il convient de mettre en balance le caractère exceptionnel de la menace d'atteinte à la sécurité publique en raison du comportement personnel de la personne concernée «à l'aune notamment des peines encourues et de celles retenues, du degré d'implication dans l'activité criminelle, de l'ampleur du préjudice et, le cas échéant, de la tendance à la récidive, (...) avec d'autre part, le risque de compromettre la réinsertion sociale du citoyen de l'Union dans l'Etat où il est véritablement intégré, laquelle est dans l'intérêt non seulement de ce dernier, mais également de l'Union européenne en général » (point 50). Au-delà donc de la marge nationale d'appréciation, c'est "l'intérêt de l'Union » qui est ici mis en exergue, le juge national devant aussi tenir compte des droits fondamentaux dont la Cour assure le respect (point 52). Sous ces conditions, le juge de l'Union considère que la lutte contre la criminalité organisée peut relever de la notion de " raisons impérieuses de sécurité publique ».

Expulser un citoyen de l'Union ayant passé légalement la majeure partie, sinon l'intégralité, de son enfance et de sa jeunesse dans l'Etat membre d'accueil ne peut être admis que si l'Etat avance "de très solides raisons pour justifier la mesure d'éloignement " (point 53). A bon entendeur...

CJUE, 23 novembre 2010, Tsakouridis, aff. C-145/09.

\section{AUTEUR}

\section{MYRIAM BENLOLO CARABOT}

Myriam Benlolo Carabot, professeure de droit privé à l'université de Nanterre, est spécialiste de droit européen (Union européenne et Convention européenne des droits de l'homme) 